\title{
Nordiques
}

\section{À l'école de l'autonomie nordique : interview collective des membres du consortium Erasmus lycée Jehan-Ango de Dieppe}

Yohann Aucante et Maria Hellerstedt

\section{CpenEdition}

Journals

Édition électronique

URL : http://journals.openedition.org/nordiques/523

DOI : $10.4000 /$ nordiques.523

ISSN : 2777-8479

Éditeur :

Association Norden, Bibliothèque de Caen la mer

\section{Édition imprimée}

Date de publication : 1 novembre 2018

Pagination : 77-97

ISBN : 9791095914020

ISSN : $1761-7677$

\section{Référence électronique}

Yohann Aucante et Maria Hellerstedt, « À l'école de l'autonomie nordique : interview collective des membres du consortium Erasmus lycée Jehan-Ango de Dieppe », Nordiques [En ligne], 36 | 2018, mis en ligne le 31 octobre 2020, consulté le 13 mars 2021. URL : http://journals.openedition.org/ nordiques/523 ; DOI : https://doi.org/10.4000/nordiques.523 


\section{À l'école de l'autonomie nordique : interview collective des membres du consortium Erasmus lycée Jehan-Ango de Dieppe ${ }^{1}$}

En 1960, Louis Legrand publie, sous le titre Pour une pédagogie de l'étonnement, la thèse qu'avait dirigée Paul Ricœur sur les Principes philosophiques d'une pédagogie de l'explicitation et qu'il avait soutenue deux ans plus tôt. En 1981, il est appelé par Alain Savary, ministre de l'Éducation nationale, pour piloter la réflexion sur la réforme du collège, devenu " unique » en 1975. Il remet son rapport en décembre 1982. Celui-ci témoigne de son souci de "faire prévaloir l'apprentissage sur l'enseignement, la promotion sur la sélection, l'autonomie sur le conditionnement, la coopération sur la compétition » (p. 9). Peu des préconisations contenues dans ce rapport seront finalement retenues par le ministre dont le départ, en 1984, signera la fin de cette période intense de réflexion pédagogique et le retour aux « fondamentaux ».

Pourtant, son successeur, en fixant à l'École l'objectif d'amener $80 \%$ d'une classe d'âge au niveau du baccalauréat, contribuera à faire entrer au lycée les problématiques que le rapport de Louis Legrand tentait d'appréhender pour le collège (gérer l'hétérogénéité des publics, susciter le désir d'apprendre...).

Sans revendiquer une quelconque filiation avec les recherches de Louis Legrand, force est de constater que le projet de l'établissement coordonnateur du consortium Erasmus et porteur du projet présenté dans l'entretien collectif qui suit tente de trouver des réponses aux mêmes interrogations, quelques-unes d'entre elles au moins.

Quant à l'étonnement (faire l'expérience d'une "insuffisance momentanée »²), si Louis Legrand le pensait comme moyen de motiver chez l'élève l'apprentissage, nous nous sommes approprié ce concept comme vecteur de professionnalisation en éveillant en nous un désir de recherche, un engagement personnel, voire une responsabilité.

Nourrie par nos lectures, notre quête nous incite à trouver des moyens de lutter contre les inégalités de toutes sortes et à la suite d'Aziz Jellab ${ }^{3}$, nous pensons que l'établissement peut être un échelon pertinent pour cela. Cet engagement est, par

1 Propos recueillis au lycée Jehan-Ango de Dieppe le jeudi 31 mai 2018.

2 Louis Legrand, Pour une pédagogie de l'étonnement, Paris, Delachaux et Niestlé, 1960.

3 Aziz Jellab, Pour un établissement équitable, Boulogne-Billancourt, Berger-Levrault, 2017. 
ailleurs, de nature à offrir une réponse aux divers malaises enseignants (sentiment d'inefficacité, de reproduction des inégalités...) bien décrits par Anne Barrèré.

Confortés par les recherches récentes en sciences cognitives, nous sommes convaincus qu'il nous faut inviter les élèves à adopter une posture réflexive, à construire leur désir d'apprendre et donc leur autonomie et à développer, dans le même temps, des expériences collaboratives. Nous nous sommes préparés à rencontrer des organisations très éloignées de nos pratiques actuelles, non pas pour les dupliquer mais pour étayer notre propre réflexion.

Celle-ci ne nous conduit-elle pas à rêver une société démocratique préfigurée par un modèle éducatif tourné vers la formation de citoyens ${ }^{5}$ ?

"Notre époque, écrivait en 1910 la grande pédagogue suédoise Ellen Key, réclame à grands cris de la personnalité, mais le fera en vain tant que nous ne donnerons pas aux élèves la possibilité d'avoir leur propre volonté, de penser tout seuls, de se constituer eux-mêmes leur propre savoir, de se former leur propre jugement ; bref, tant que nous ne cesserons pas de réprimer dans les écoles la matière brute de la personnalité en espérant vainement qu'elle ressuscite plus tard. "

Nous étonner : pourquoi nos collègues des pays visités ne pratiquent-ils pas comme nous? Prendre des initiatives, voire des risques (calculés) : expérimenter de nouveaux dispositifs pédagogiques valorisant les talents des élèves inspirés par les analyses que nous ferons des différences observées. Faire des émules : partager nos découvertes en permettant aux enseignants français de cheminer vers elles d'une manière isomorphe à celle que nous souhaitons promouvoir pour les élèves.

Voilà le triple défi que nous nous sommes fixé.

Dominique Procureur, proviseur et Audrey Techer, professeur de philosophie

Yohann Aucante: Quelles circonstances, quels constats ou objectifs ont mené à l'initiation de ce programme?

Dominique Procureur (proviseur du lycée Jehan-Ango de Dieppe et chef de projet ainsi que chef du consortium Erasmus qui l'a porté, membre de la délégation finlandaise) : À l'origine du projet, il y a le travail que ce lycée avait entamé pour écrire le projet d'établissement (qui est une obligation réglementaire). Au cours de son écriture, nous avons centré notre réflexion sur la question des apprentissages : comment faire en sorte d'améliorer les techniques d'apprentissage

4 Anne Barrère, Au coeur des malaises enseignants, Paris, Armand Colin, 2017.

5 Le rapport remis par Louis Legrand à Alain Savary en 1982 s'intitulait Pour un collège démocratique.

6 Ellen Key, Le siècle de l'enfant, Paris, Flammarion, 1910, cité par Philippe Meirieu dans Louis Legrand (1921-2016). Pédagogie et politique, Paris, L'Harmattan, 2018. 
des élèves ? Dans un premier temps, on a créé des groupes qui ont réfléchi à cette question et on s'est demandé ce qui pouvait constituer des freins à cet apprentissage puisque nous n'étions pas satisfaits du résultat auquel aboutissaient les élèves. De là, on a exploré plusieurs pistes : l'une d'entre elles était ce que pouvaient nous apporter les sciences cognitives et ce travail se poursuit en parallèle dans le lycée ; et à un moment donné, on s'est dit " et si on allait voir ailleurs ?». On sait qu'il y a des endroits où il $\mathrm{y}$ a une plus grande satisfaction au niveau des résultats obtenus. J'ai donc commencé ce travail d'exploration un peu au hasard, en expliquant quelles étaient nos préoccupations et en demandant aux enseignants qui répondaient à ces critères de se manifester. Cela a duré presque six mois sans réponse. Il ne s'est rien passé en utilisant les outils à notre disposition : e-twining, la délégation académique aux relations internationales... J'ai tapé à plusieurs portes sans obtenir de réponse. Quelques mois plus tard, alors que j'avais presque abandonné, je suis tombé sur un article dans une revue qui parlait de la question de l'évaluation et de la motivation qui croisait nos préoccupations. J'ai pris contact avec l'auteur de l'article, par ailleurs associé au Centre international d'études pédagogiques (CIEP) de Sèvres, organisme qui fait venir les assistants étrangers en France et organise le déplacement des assistants français à l'étranger. Je me suis dit que cette personne devait avoir des relations à l'extérieur et que par son intermédiaire, on pourrait vraisemblablement entrer en contact avec les pays qu'on visait. Ce qui a été le cas puisqu'elle nous a apporté trois partenaires. Et parallèlement, par le biais de relations personnelles, Audrey Techer s'est trouvée en contact avec des collègues d'autres pays. On s'est retrouvé, en l'espace de trois semaines, avec cinq partenaires : trois en Scandinavie, un au Canada et un en Australie. Cela nous a un peu effrayés, car on se demandait si on allait être capable de gérer autant de liens, mais on s'est lancé : on s'est réuni ici, on s'est réparti les pays, il y avait un interlocuteur pour chacune des destinations. On s'est aussi posé la question de savoir si on faisait cela tout seuls ou si cela n'avait pas plus de sens de s'occuper de toute la chaîne, de l'enfant lorsqu'il entre à l'école jusqu'à l'université. C'est cette option que nous avons choisie, et donc l'idée de ce consortium européen, avec des professeurs d'école, de collège, et de lycée. Initialement, on avait prévu également des inspecteurs pour qu'eux aussi puissent avoir leur regard spécifique sur ce qu'on allait observer et des personnels de direction pour la dimension établissement tout entier. Depuis nous avons perdu un partenaire : l'Australie ne répond plus. Nous n'avons pas pu intégrer le Canada, car les crédits que nous allouait l'Europe ne concernaient que les pays européens. J'ai commencé à construire mon budget et collecter quelques fonds pour le Canada, mais il reste à convaincre quelques collectivités territoriales de contribuer afin que nous puissions aller aussi à Québec, à Montréal plus exactement où se trouve notre partenaire. 
Yohann Aucante: Y a-t-il eu d'autres acteurs qui ont contribué à impulser ou à accompagner ce programme en le considérant comme important ?

Dominique Procureur : J'avais également et initialement pensé que l'ESPE (École supérieure du professorat et de l'éducation) devait jouer un rôle, mais pour la même raison que pour les inspecteurs nous n'avons pas pu les intégrer. Il s'agit d'un problème de positionnement hiérarchique : le lycée étant la tête du consortium, nous n'avons pas réussi à intégrer des personnels qui n'étaient pas dépendants de l'établissement parce que, hiérarchiquement parlant, il n'était pas pensable qu'une inspection académique ou un rectorat devienne un membre d'un consortium piloté par un établissement, ce qui aurait inversé les normes. On n’a pas non plus eu le temps d'intégrer l'ESPE ${ }^{7}$ parce qu'il aurait fallu passer une convention avec celle-ci, mais elle travaille encore avec nous. On a écrit le projet à 25 mains. En cours de route, on a continué à chercher, à faire des lectures. La délégation académique aux relations internationales de Rouen nous a aussi aidés, y compris durant la phase d'écriture.

Yohann Aucante: La Scandinavie a-t-elle été une cible consciente puisque vous dites qu'il y a eu recherche dans différentes directions et d'autres pays comme le Canada? Quelles représentations aviez-vous de ces pays par rapport à ces logiques d'apprentissage?

Éric Lelong (principal du collège de Cany-Barville, membre de la délégation suédoise) : La représentation que j'avais de la Suède et des pays scandinaves, c'était une représentation par le biais de ce qu'on peut lire dans les revues notamment sur PISA et le système éducatif finlandais et scandinave en général, donc une représentation avant tout de réussite en termes de système éducatif et dont on pouvait retirer des choses intéressantes pour le système français.

Yohann Aucante : Pourtant avec une grande variabilité des résultats de PISA. Quand on regarde les résultats, la France n'est pas si mal placée par rapport au Danemark et à la Suède.

Éric Lelong : Oui, la lecture de PISA peut être variable selon là où l'on se place. On ne peut nier que dans le système éducatif français et selon le ministère notamment, PISA est un outil très fortement utilisé pour mener les politiques éducatives en France.

Yohann Aucante : Le choix des pays scandinaves était-il simplement lié aux pays qui ont pu répondre ou est-ce que c'était vraiment l'objectif d'aller voir en Scandinavie?

7 École supérieure du professorat et de l'éducation. 
Dominique Procureur : Ce qu'on cherchait à voir, c'était des élèves qui travaillaient en autonomie, notre recherche était en premier lieu orientée par cette nécessité. Et les pays que nous avons sélectionnés, qui nous ont été proposés, le sont au travers de ce que nous avons communiqué de notre intention. Peutêtre que si on avait affiché que l'on cherchait autre chose se serait-on dirigé vers d'autres destinations que celles-là. Par ailleurs, même si nous n'avions pas une idée très précise des différences (que nous connaissons maintenant), ces pays qui nous étaient proposés correspondaient vraisemblablement à une représentation que nous avions de quelque chose qui marche, d'endroits où l'on accepte de faire des expériences. Contrairement à ce qui se passe en France, là-bas il y a des rapports qui sortent et qui sont utilisés, ils ne restent pas dans des tiroirs, on essaie des choses et, si on s'aperçoit que ça ne marche pas, on accepte assez aisément de revenir en arrière et puis de reconsidérer la situation.

Yohann Aucante : Était-ce une représentation partagée par tout le monde lorsque vous avez travaillé à la construction de ce projet ?

Béatrice Vallée (principale adjointe du collège Rachel-Salmona du Tréport, membre de la délégation danoise): Ce qui était important, c'était l'autonomie, mais également ce qui était important dans les représentations, c'était la notion de " climat scolaire » et de bien-être à l'école, car on a l'impression que ce sont des pays où aller à l'école est facile, que l'enfant va y aller plus facilement qu'en France.

Murielle Bonnoron (professeur de mathématiques au collège Rachel-Salmona, membre de la délégation suédoise) : Je suis pour ma part enseignante au collège et cette opportunité nous a été proposée après la mise en place du projet par notre chef d'établissement qui nous a expliqué de quoi il retournait et je trouvais intéressant d'aller voir dans d'autres pays les pratiques pédagogiques pour permettre aux élèves d'accéder à davantage d'autonomie.

\section{Florence Lenois (principale adjointe du collège Albert-Schweitzer de Neufchâtel-} en-Bray, membre de la délégation suédoise) : c'est la même chose pour moi, le projet était très avancé lorsque j’y suis entrée. Mais je l'ai trouvé très intéressant, car j'avais une représentation de ces pays comme étant très performants sur le plan éducatif. Et je suis partie avec l'idée de travailler sur la notion d'autonomie, car c'était la base du projet du lycée Ango, mais je m'étais dit qu'il y aurait sans doute des choses transférables au collège. Et c'est vrai que, par rapport au climat scolaire, il y a des choses à mettre en place dès le primaire concernant la bienveillance, le respect physique des jeunes. Et j'ai été ravie de participer à cette expérience.

Dimitri Vaultier (professeur d'anglais au collège Albert-Schweitzer, membre de la délégation suédoise) : Moi aussi je suis arrivé a posteriori, le choix des pays était déjà fait, mais c'est un choix qui m'a paru tout de suite cohérent par rapport à l'imaginaire que je pouvais avoir de ces pays-là, et également dans mon entourage. 
En effet, en tant qu'enseignant, on peut avoir une certaine représentation, mais pour en avoir parlé autour de moi, pour avoir dit que je partais avec des collègues étudier l'autonomie, le bien-être des élèves dans les pays scandinaves, ça a paru cohérent à tout le monde, plus que si l'on était parti en Espagne ou en Pologne, même si cela aurait été certainement très intéressant également.

Dominique Procureur : Nous avons travaillé avec trois collèges, nous avons monté le projet avec trois principaux, dont deux qui ont changé d'affectation. Par exemple, Éric Lelong est arrivé en septembre dans son établissement et cela n’a pas arrêté le processus. Il s'est très vite engagé jusqu’à se déplacer en Suède. Ça a été le cas aussi pour l'établissement de Neufchâtel-en-Bray où le chef d'établissement a changé en septembre, donc celui qui a écrit une partie du projet est maintenant parti loin d'ici et pour autant le collège a continué à travailler. Cela signifie que les choses étaient suffisamment installées et réfléchies pour que cela continue malgré les changements de responsable à la tête des institutions qui étaient parties prenantes.

Yohann Aucante : Du côté scandinave, avez-vous essayé de viser différents secteurs, différents types d'établissement?

Estelle Bucquet (professeur d'anglais au lycée Jehan-Ango, membre et chef de la délégation danoise) : Pour le Danemark, on est parti d'un correspondant français. Il avait une grande expérience du travail en France et au Danemark, et c'est avec lui qu'on a communiqué pour savoir si on pouvait voir différents niveaux et c'est lui qui nous a aidés à établir des contacts avec un autre établissement. Grâce à lui, on a pu établir des liens avec celle qui est devenue aujourd'hui notre référente sur place et qui est au lycée.

Amélie Crespe (professeur d'anglais au lycée Jehan-Ango, membre et chef de la délégation finlandaise) : Pour la Finlande, nous avions un contact qui est enseignant dans un lycée professionnel (ou plutôt lycée des carrières, car il y a aussi la formation continue), et qui nous avait été recommandé par Audrey Techer, collègue de philosophie au lycée Ango. Il nous a demandé ce qu'on voulait voir étant donné que nous n'étions pas dans le même type d'établissement et n'avions pas les mêmes priorités et c'est lui qui s'est arrangé pour trouver différents types d'établissements : on a donc visité le jardin d'enfants, l'école primaire, le collège, le lycée général, le lycée professionnel. On a même pris des contacts avec l'université la plus proche. Mais sur la zone géographique, on était très limité, on était dans les provinces intérieures de la Finlande. On a toutefois vu des profils variés, avec du rural et du semi-urbain. Mais dans l'école primaire que l'on a visitée, il n'y avait que 42 élèves scolarisés. C'était la toute petite école dans la forêt !

Audrey Techer (professeur de philosophie au lycée Jehan-Ango, membre et chef de la délégation suédoise) : Pour la Suède, nous avons vécu une épopée. Au départ, 
on n'a pas été sélectif, car on était tellement en demande de partenaire qu'on a accepté les contacts qu'on a eus. Notre premier contact était le lycée professionnel du Munkagårdsgymnasiet de Tvååker et il s'est produit un gros malentendu qui a fait que ce partenaire nous a laissés tomber au mois d'octobre précédant notre voyage ; il a donc fallu en trouver d'autres au pied levé. Sur Internet, j’ai trouvé un lycée privé (Mikael Elias teoretiska gymnasium) à Göteborg qui a accepté de nous prendre comme partenaire avec beaucoup d'enthousiasme. Par ailleurs, j'avais envoyé un mail au grand lycée public de Varberg (Peder Skrivares skola), mais la proviseure n'avait trouvé aucun enseignant intéressé par notre projet. Par un autre biais, j'ai pu avoir deux contacts à Varberg, dans ce même lycée, qui nous ont répondu avec enthousiasme et qui nous ont accueillis. Et après, il s'est trouvé que le collègue qui nous avait fait faux bond est revenu proposer non seulement de nous accueillir, mais aussi de nous faire visiter un autre établissement, l'Ankarskolan de Träslövsläge. Par ailleurs, un ami des contacts de Peder Skrivares enseignait à la Franska skolan de Göteborg. Nous avons ainsi eu l'opportunité de voir cinq établissements différents, deux publics et trois privés ; on a fait tous les niveaux, depuis l'école fondamentale jusqu'au lycée ; urbains et ruraux. Mais du coup on a eu des sons de cloche très différents, ce qui m’a personnellement déstabilisée. Ce n'est pas plus mal, mais c'est par conséquent difficile de se faire une opinion définitive et monolithique du système éducatif suédois.

Éric Lelong $\left(S^{8}\right)$ : Juste pour préciser, on a vu du rural ou semi-rural et de l'urbain. On s'est posé la question de la représentativité sociale de ce que l'on voyait, car on avait l'impression d'avoir plutôt un niveau social élevé dans les établissements qu'on a visités. On pense ne pas avoir vu d'établissements dans des zones urbaines plus difficiles.

Florence Lenois $(S)$ : Cela apparaissait dans le climat scolaire qui était apaisé. On n'a pas vu de bousculade, on n'a pas été témoins de bagarres, de tensions, ce qui nous fait dire que la population était assez « éduquée ». En milieu rural la situation avait l'air favorisée, car c'était de plus petits effectifs, les espaces étaient grands. On a vu des établissements où les couloirs étaient grands et où l'on pouvait tous se déplacer sans se heurter. Il y a beaucoup de facteurs qui nous ont amenés à nous demander si l'on pouvait généraliser ce qu'on a vu à toute la Suède.

Séverine Caron (professeur des écoles à Envermeu, membre de la délégation finlandaise) : C'est la même chose pour les autres délégations. On a vu un certain nombre d'écoles et d'établissements et l'on ne peut généraliser. Si l'on venait observer en France, on ne verrait de la même façon que quelques classes dans un paysage très diversifié.

8 Pour plus de commodité, les initiales près des noms renverront au pays visité : « $S$ " pour Suède, « $\mathrm{D}$ » pour Danemark et « $\mathrm{F}$ » pour Finlande. 
Yohann Aucante : Pourriez-vous donner quelques éléments sur la manière dont vous avez préparé le séjour?

Estelle Bucquet (D) : On a commencé par travailler ensemble sur l'élaboration de grilles - dont on s'est ensuite détaché très vite, mais qui nous ont vraiment aidés parce qu'elles ont constitué la base des pistes de réflexion. Surtout, on les a testées en allant observer dans les établissements français des cours de disciplines dont on ignorait tout et c'est là que l'on s'est aperçu qu'on n'avait pas envie de se limiter à un cadre (même s'il nous guidait) et qu'on avait besoin de sortir de ces grilles. Donc on a travaillé différemment : on a fait des binômes (on était 6) et on a choisi de grands axes d'observation et tous les soirs on faisait le bilan, on mettait en commun, on commençait à synthétiser et on réfléchissait aux questions pour le jour suivant.

Murielle Bonnoron (S) : Cela vaut pour le Danemark et vous alliez voir les classes tous ensemble alors qu'en Suède ce n'était pas le cas : nous n'étions pas ensemble dans les observations des classes, nous étions soit en binômes, soit en trinômes donc il n'était pas possible de se répartir des axes à observer. Nous regardions donc un petit peu tout. Et on a fait nos bilans après.

Florence Lenois $(S)$ : Je voudrais souligner le travail qui a été fait en amont par toute l'équipe, mais en ce qui nous concerne, particulièrement par Audrey Techer, qui nous a donné une "bible ». On avait un document comme celui-ci [elle montre un petit dossier A4 relié] avec le parcours, les établissements, les personnes qu'on allait rencontrer avec leurs photos ; je pense que cela a facilité les échanges dès le départ.

Florence Bouteiller (professeur d'anglais au lycée Jehan-Ango) : Au niveau de la préparation, on n'a pas fait que des grilles ; on a essayé d'anticiper ce qu'on pourrait voir. Et pour cela, certains (délégations danoise et finlandaise) sont allés au Département des études nordiques de l'université de Caen assister à une conférence. Sur la Suède il n'y avait rien, d'où la rencontre d'aujourd'hui avec vous. On avait également créé un espace virtuel de partage dans lequel on pouvait déposer ou lire les documents que les collègues avaient déposés afin d'avoir des informations sur la société du pays dans lequel on allait, sur le système politique, éducatif afin de ne pas arriver vierges et tout découvrir sur place, ce qui aurait été impossible. Une autre chose que l'on a faite, c'est de proposer une formation de remise à niveau en anglais pour les collègues qui ne se sentaient pas sûrs d'eux. On s'est basé sur des documents qui parlaient du système éducatif de ces pays en langue anglaise afin de maîtriser un peu de vocabulaire et d'être à même de poser des questions à nos interlocuteurs locaux.

Audrey Techer (S) : Je rajoute qu'on a reçu ici la visite de Roger-François Gauthier, qui est le directeur de la Revue internationale d'éducation de Sèvres, et 
de sa doctorante, Maria Lombardi. Ils nous ont proposé deux interventions très intéressantes sur la méthodologie de la comparaison des systèmes éducatifs en Europe. Cela nous a bien préparés intellectuellement à nos observations.

Estelle Bucquet (D) : Pour le Danemark, on a reçu une aide précieuse en la personne de Lise Roelandt, notre collègue d'origine danoise qui est professeur d'EPS au collège de Cany-Barville, dont la mère est danoise et qui nous a bien renseignés sur la culture du pays, qui nous a aussi donné des pistes de lecture, prêté des livres.

Véronique Caijo (proviseur adjoint du lycée Jehan-Ango): Avant chaque départ de délégation et à chaque retour de délégation, il y a eu débriefing avec l'ensemble de l'équipe. Les collègues ont eu des temps d'échange sur l'expérience pendant leur séjour, ils ont dit ce qui avait fonctionné ou pas, comment ils s'étaient organisés, ce qu'il était utile de faire ou de ne pas faire, etc., de façon à ce que la délégation suivante qui allait partir ait quelques idées pour leur voyage. Ça a été précieux pour les deux délégations qui sont parties après la première. Systématiquement, on a favorisé ces temps d'échange, qui permettaient de prolonger la soirée et de continuer la discussion sur ce qui avait été vécu. Et dans les discussions qu'on a eues sur les retours de délégations, on a aussi eu des réflexions qui portaient sur ce qu'il était possible ou non d'adapter de ce qui avait été observé, dans la pratique quotidienne. Cela a suscité une vraie interrogation sur nos structures, nos locaux, sur ce qu'on pourrait mettre en place. On a déjà commencé à faire des choses dans l'établissement, au sujet du mobilier, qu'on ne trouve pas adapté. Il faudrait peut-être réfléchir à l'organisation de nos classes. On s'est aussi aperçu qu'on ne pourrait pas faire certaines choses, car notre système (règlement intérieur, notre fonctionnement) nous contraint, mais que pourtant on pourrait trouver quelques astuces pour que ça fonctionne mieux avec les élèves. L'échange de pratiques entre nous a créé une certaine solidarité, et même de l'amitié. On a appris à se connaître en inter-niveaux.

Éric Lelong (S) : Je trouve que c'était intéressant que dans chaque délégation il y ait une représentativité large des personnels : des enseignants de différentes disciplines, des personnels du premier et du second degré, de direction ; cela permet des points de vue et des entrées diverses qui enrichissent les observations.

Nicolas Buquet (professeur des écoles et directeur de l'école Pierre-Curie à Neuvilleles-Dieppe, membre de la délégation danoise): Cela les a enrichies d'autant plus que cela a permis de voir que le premier degré peut se tourner vers le second degré, qu'on n'est pas si éloigné que ça lorsqu'on se met à travailler ensemble; on a l'impression de deux mondes parallèles, mais on peut agir et aller dans la même direction. De mon point de vue, dans le premier degré, ça a été très riche pour ça.

Yohann Aucante: Qu'est-ce qui vous a frappés quand vous êtes arrivés sur place, concernant l'environnement de travail par rapport à ce que vous connaissez ? 
Murielle Bonnoron (S) : En Suède, les cours n'ont pas tous la même durée : certains vont durer 30 minutes, d'autres 1 heure, d'autres 80 minutes... et il n'y a aucune sonnerie, ce qui est assez surprenant. Et la circulation se fait naturellement, elle est fluide, même si ce n'est pas les mêmes horaires pour tout le monde, ça ne gêne personne.

Dimitri Vaultier $(S)$ : Ce qui nous a frappés en Suède et qui est certainement commun à toutes les délégations, c'est qu'ils ont beaucoup moins de règles qui pourraient paraître secondaires, de type enlever son manteau, demander à se lever pour aller à la corbeille, demander pour ceci et cela, c'est-à-dire moins de règles qui sont purement et simplement contraignantes, ce qui à mon avis permet un épanouissement plus important des élèves et qui fait qu'ils se sentent moins dans un carcan quand ils arrivent à l'école.

Yohann Aucante : Là, il y a une source claire d'autonomie donc?

Dimitri Vaultier (S) : Certainement. Cela dit, s'agissant de mon expérience personnelle et pour en avoir discuté avec des collègues qui sont d'accord avec moi, j'ai tendance à essayer de mettre moins de règles dans ma classe ; par exemple, je passe mon temps à leur dire : "Ne me demande pas pour aller à la corbeille, tu me coupes la parole, ça ne sert à rien, vas-y, tu ne demandes pas chez toi pour aller à la poubelle. » Mais si sur l'ensemble des élèves, c'est plutôt quelque chose de positif, il y en a malgré tout certains pour qui ça devient un jeu de contourner ce qui habituellement est une barrière. Et ce qu'on s'est tous dit, c'est que tant qu'on ne fonctionnera pas tous sur un modèle similaire, c'est-à-dire avec moins de contraintes, ça restera contre-productif.

Florence Bouteiller : J'interviens en tant que personne qui n'est pas partie. S'agissant des trois délégations, ce que j'ai davantage noté, c'est que vous avez été choqués par le fait que les élèves investissent l'espace de manière très libre par rapport à chez nous. Chez nous, soit ils sont assis là où on leur dit, soit on leur dit de faire un groupe de trois ou de quatre et on les dispose dans la classe et ils restent dans la classe, alors que là tous étaient surpris par le fait que les élèves puissent décider de l'endroit où ils allaient travailler. On leur donne une tâche à accomplir et ils vont l'accomplir là où ça leur paraît le plus agréable ou le plus propice.

Florence Lenois $(S)$ : C'est vrai que si on veut changer les règles, il faut qu'il y ait une cohérence entre les différents intervenants ; un enseignant ne peut pas se dire " moi je vais fonctionner comme ça ", en étant en marge de la façon de faire des autres collègues : ça ne peut pas marcher. Et il faut aussi qu'il y ait une continuité primaire, collège et lycée. Pour citer un exemple : on a échangé avec Maria Hellerstedt concernant le tutoiement. On a vu là-bas tous les élèves tutoyer les adultes qu'on a rencontrés. Pour eux, c'est naturel, c'est fait depuis le primaire, ce n'est pas un manque de respect, ce n'est pas de la familiarité. En France, nous a dit 
Maria Hellerstedt, ça se passait bien la plupart du temps, mais elle devait remettre les règles en place pour certains en expliquant que le tutoiement ne signifiait pas qu'elle était leur " copine ». J'ai aussi pris des photos d'élèves qui avaient les pieds sur la table pour citer un autre exemple, mais ils travaillaient et ils étaient en chaussettes donc ils n'abîmaient pas le mobilier. Ils étaient " avachis » dans un fauteuil, avec les pieds sur la table et ils travaillaient. J'ai montré ces photos à ma fille qui va au collège pour lui prouver qu'il y avait moins de contraintes physiques en Suède et elle m’a répondu : "si nous, on faisait ça, ce serait vite le bazar ", car les élèves iraient bien au-delà des seuls pieds sur la table et on ne serait plus dans un contexte de travail. D'où la nécessité que tout se mette en place dès le début et qu'il y ait une continuité. C'est pour cela aussi que c'était important qu'il y ait tous les niveaux (primaire-collège-lycée) représentés dans les délégations.

Nicolas Buquet (D) : En effet, dans le premier degré c'est beaucoup plus simple, j'ai déjà modifié les pratiques puisque je suis seul à avoir mes élèves toute l'année, toute la journée, toutes les semaines. Les établissements étant plus petits, on a au maximum 10-11 collègues, c'est peut-être un peu plus simple de mettre de nouvelles règles en place. J'ai aussi été surpris par tout ce qu'on peut faire dans ces pays-là - en l'occurrence au Danemark où je suis allé - où on a pensé que l'on peut agir sur l'espace et le temps des élèves pour faire en sorte qu'on n'ait pas à dépenser beaucoup d'énergie en classe à faire de la discipline, poser des règles, des contraintes. L'aménagement du temps et de l'espace fait qu'on a agi avant et que des règles sont établies; de ces règles découlent des attitudes quasiment naturelles. Les enfants, parce qu'ils ne sont pas contraints toute la journée comme disait Dimitri, font les choses avec plus de naturel.

Virginie Delaunay (directrice et professeur des écoles, à l'école de Berneval-le-Grand, dans la commune de Petit-Caux, membre de la délégation suédoise): Un gros travail a été mené sur l'architecture des établissements qu'on a visités pour le bien-être de l'élève qui va fréquenter les lieux : des couloirs qui sont immenses, spacieux dans certains établissements, des salles des profs qui sont accueillantes où le professeur peut passer une partie de la journée avec du mobilier confortable, et également dans les salles de classe. Tout est pensé pour le bien-être de l'élève qui n'a pas à s'agiter sur une chaise en bois.

Yohann Aucante: Donc en général le mobilier est accueillant et adapté ?

Florence Lenois $(S)$ : Oui, et les chaises ont des barreaux qui permettent aux élèves de reposer leurs pieds et dont on peut changer la hauteur selon l'âge de l'élève. C'est un confort important.

Hélène Perez (professeur d'anglais au collège Albert-Schweitzer de Neufchâtelen-Bray, membre de la délégation danoise) : Je suis revenue du Danemark avec plein d'idées de choses à faire et quand je suis rentrée dans mes classes et que j'ai 
expliqué ce que j'avais vu et que je voulais essayer des choses pour mes travaux de groupe, je me suis aperçue que le côté culturel était très important et que le Français aime ne pas respecter les règles. D'où l'importance de les ancrer dès le primaire.

Maria Hellerstedt: Au lycée où je travaillais à Stockholm, nous, les enseignants étions en conflit avec la direction, car on voulait qu'ils laissent tranquilles les élèves portant des casquettes. Nous considérions que ça ne les rendait pas mauvais de porter des casquettes; on voulait les laisser faire afin de se concentrer sur autre chose et cela a duré des mois avant que la direction comprenne que c'était sérieux et que l'on ne voulait pas se disputer pour cela avec les élèves.

Murielle Bonnoron (S) : C'est une histoire d'habitudes et de culture, mais depuis la réforme 9 (donc depuis l'année dernière), j'ai disposé ma classe de manière à ce qu'ils soient systématiquement en groupes ; j'avais déjà essayé de bouger mon mobilier avant la réforme, donc là c'est la deuxième année. En fait, les élèves y prennent goût et ils s'y habituent. Ce n'est donc pas seulement une question de culture, mais aussi d'habitude. Quand ils y sont habitués, ça se passe mieux.

Hélène Perez (D) : Le problème ce n'était pas le groupe pour moi, mais le respect des règles. Par exemple, je leur expliquais que, pour travailler en groupe, ils avaient le droit d'aller dans le couloir ; et ils disaient : "Ah bon, on a le droit d'aller dans les couloirs ? Oh, c'est pas intéressant alors. " En fait ce qu'ils aiment, c'est transgresser et justement ne pas respecter les règles. Il faut donc toujours qu'ils soient encadrés de règles, car sinon ils sont perdus.

Florence Lenois (S): Au collège, c'est aussi l'adolescence, dont le propre est de contester les règles établies.

Hélène Perez (D) : Oui, mais on n'a pas eu cette sensation qu'ils étaient en conflit là-bas ; justement c'est cette relation de confiance qu'on a appréciée aussi ; lorsque le professeur dit "vous avez 10 minutes ", les élèves reviennent au bout de 10 minutes sans que le professeur ait besoin de battre le rappel dans le couloir.

Florence Lenois (S) : c'est aussi l'autonomie au travail dont on parlait depuis le début.

Véronique Caijo : C'est déstabilisant, y compris pour les équipes de direction. Au retour du Danemark, un jour je vois plein d'élèves par groupes de trois avec des fiches dans un couloir : mon réflexe naturel de personnel de direction serait de les alpaguer en leur demandant ce qu'ils font là. Mais en l'occurrence je ne dis rien, je prends sur moi, je vais jusque dans une salle où je vois des élèves en train de discuter, travailler, certains debout, d'autres assis sur les tables. Et pas de prof!

9 Réforme du collège de 2016, mettant en place, entre autres, l'évaluation par compétences - plutôt que sous forme de notes chiffrées - et préconisant le travail de l'enseignant en petits groupes d'élèves. 
Je me retourne : le prof arrive, $\mathrm{M}^{\mathrm{me}}$ Bucquet en l'occurrence, qui me déclare tout de go, pour se dédouaner : «non, non ils travaillent », ce que j'avais bien compris. Mais on peut être déstabilisé par ce genre de choses. Je comprends tout à fait que les collègues peut-être un peu plus stricts ne permettent pas ce genre de choses ni ne les comprennent. Alors l'exercice consistait à préparer un oral ; ils se sont ensuite tous rassemblés dans la salle. Il faut aussi savoir accepter tout cela et c'est compliqué. Mais une fois qu'ils ont intégré ce qu'ils ont le droit de faire dans certains espaces, ça ne leur pose en effet aucun souci.

Florence Lenois $(S)$ : On l'a vu tout à l'heure quand on a traversé le hall aux portraits (du lycée Ango), il y a des petits bancs, des chaises et c'est des lieux investis par les élèves ; je n'ai pas suffisamment regardé pour voir s'ils travaillaient ou pas, mais ce sont des espaces qu'ils investissent et ils pourraient y travailler aussi.

Véronique Caijo : Pour répondre sur ce point, on mène aussi un projet sur l'aménagement des espaces dans l'établissement avec les élèves. On a contacté Canopé ${ }^{10}$ qui nous a aidés durant une journée où ils ont organisé un " créathon ». On avait préparé cette action-là avec eux ; ils ont exploré les endroits que les élèves investissent $-\mathrm{M}^{\mathrm{me}}$ Techer a travaillé d'ailleurs avec un groupe. Sur cette journéelà, ils ont fait des propositions d'aménagement et l'objectif était : « Lâchez-vous ! C'est votre établissement scolaire, faites des propositions d'aménagement pour bien y vivre. "Quand on les regarde déambuler dans un établissement scolaire, ils investissent les sous-escaliers, les petits coins, les bancs qui sont à l'intérieur du hall mais pas forcément de façon orthodoxe, c'est-à-dire qu'au lieu de s'asseoir, ils se couchent, ils inversent le banc et ont les pieds qui pendent à l'intérieur, etc. Notre réflexe est alors de les reprendre pour les remettre dans le bon sens, mais pour eux c'est une question d'échange. Et la dernière fois, ils étaient inversés et discutaient entre eux, car le haut du banc leur servait de tablette où ils pouvaient poser leurs cahiers. Mais en fait ils discutaient. Ils ont donc détourné du mobilier qui était installé dans l'établissement pour échanger. La question qui se pose alors est : "Que fait-on de notre mobilier ?"

Estelle Bucquet (D) : C'est exactement la même chose que ces élèves dans les couloirs au sujet desquels l'impression première de n'importe quel français serait que c'est le bazar. Finalement, comme l'a confirmé la collègue qui était dans la salle à côté de moi, ils ne gênaient pas du tout. Mais je me suis moi-même posé la question avant de tenter cela parce qu'on a l'habitude de faire des travaux de groupe, mais de là à les laisser investir l'espace, c'est quelque chose d'innovant. Et l'une des raisons pour lesquelles j'ai fait ça, c'est parce que c'est une très bonne classe (euro), mais dans laquelle les élèves n'ont pas du tout confiance en eux

10 Canopé est un réseau de création et d'accompagnement de ressources pédagogiques, placé sous la tutelle du ministère de l'Éducation nationale, qui édite des ressources transmédia répondant aux besoins de la communauté éducative et qui a vocation à faire entrer l'École dans l'ère numérique. 
parce qu'ils passent leur temps à se comparer aux autres. Or, la chose qui m’a le plus frappée au Danemark, c'est justement la perception de cette confiance en soi parce qu'on leur fait confiance, on les responsabilise et on sent bien que les élèves ont confiance en eux parce qu'ils ont l'habitude qu'on les laisse travailler en petits groupes, qu'on leur donne des tâches, qu'on leur donne un temps déterminé pour les faire. On a vu une enseignante travailler avec une petite cloche et à la fin du temps imparti, les élèves reviennent avec le travail fait en bonne et due forme et il n'y a ni stress ni pression. Le simple fait de faire à l'extérieur de la classe ce que j'aurais pu faire à l'intérieur, c'est-à-dire de permettre à l'élève de sortir, ça a eu comme résultat que tous les élèves ont fait ce que je leur avais demandé, sans avoir peur de se faire écouter par de très bons élèves anglophones.

Dimitri Vaultier $(S)$ : Je reviens sur ce que tu [V. Caijo] disais, à savoir qu'il faut que le personnel de direction prenne l'habitude et s'adapte, c'est valable pour nous les enseignants, mais également pour les élèves. Par exemple, pour ma part, cette semaine, j'ai eu une classe de $4^{\mathrm{e}} \mathrm{SEGPA}{ }^{11}$ qui est arrivée mardi soir après six heures d'atelier, épuisée, dans une salle où il faisait 25-30 degrés, en ronchonnant et en déclarant ne pas avoir envie de travailler et en proposant, sur le ton du défi, de faire cours dehors. Ce à quoi j'ai dit d'accord, en leur demandant d'emporter leurs dictionnaires, un crayon et du papier et en leur proposant d'aller s'asseoir par terre dans la cour pour travailler. Ils sont tombés des nues. Or, il s'est avéré qu'on a travaillé, ils ont fait une bonne séance, ce qui n'est pas toujours le cas, surtout le mardi en fin de journée, mais il y a eu deux minutes pendant lesquelles ils étaient très angoissés à l'idée que la principale passe et les dispute. Il a fallu que je les rassure.

Yohann Aucante : De quelle manière ou sur quels points ces séjours ont-ils confirmé ou déstabilisé vos opinions sur les systèmes éducatifs de ces pays et sur leur réussite?

Séverine Caron $(F)$ : Pour la Finlande, on a vu un système qui fonctionnait très bien, mais c'est culturel et lié au mode de vie et nous avons tous constaté qu'on n’avait pas observé de méthode pédagogique extraordinaire. Beaucoup de travaux de groupe, certes, mais on n'a rien vu de très différent de ce qui se fait en France, ce qui nous a beaucoup déstabilisés.

Nicolas Buquet (D) : En ce qui me concerne, ça a confirmé chez moi le droit d'être déstabilisé et j'assume de l'être désormais. Le regard de mes collègues, des familles, me pose beaucoup moins de problèmes. Je me sens le droit de tenter des choses, qui fonctionnent ou qui ne fonctionnent pas. Ce n'est pas grave, j’ai tenté,

11 Au collège, les sections d'enseignement général et professionnel adapté (SEGPA) accueillent des élèves présentant des difficultés scolaires graves et persistantes auxquelles n'ont pu remédier les actions de prévention, d'aide et de soutien. 
j'explique et du coup on peut essayer d'avancer de cette manière-là. Et ça déstabilise aussi les élèves comme l'a dit Dimitri. J'ai des élèves qui me disent : "C'est vrai, vous n’allez rien dire ? " À quoi je réponds, "ben non, ce n’est pas grave, tu t'es mal comporté, on va en discuter ". J'ai un peu mis de côté le regard et le jugement de mes collègues et de l'institution. C'est peut-être plus simple d'assumer parce que je commence à avoir un petit peu d'expérience, mais je crois qu'il faudrait peut-être dans la formation s'inspirer de cela et dire aux jeunes collègues de se lancer et vous verrez avec le recul si c'était bien ou pas bien.

Florence Lenois (S) : Pour la Suède, tout n'est pas transposable, par exemple, par rapport à la pédagogie, j'ai assisté à quelques cours de français où je me suis dit que j'aurais adoré apprendre l'anglais comme cela, même si beaucoup de choses ont dû évoluer depuis l'époque où j'étais élève. Mais j'ai beaucoup apprécié les séquences de 10 minutes, les travaux en duo ou petits groupes; cette alternance toutes les 10 minutes sollicitait vraiment l'attention des élèves. Après, en discutant avec les collègues, j'ai appris qu'il y avait beaucoup de choses qui se faisaient aussi en France. Mais la marge de progrès peut être sur la bienveillance et la confiance que les élèves peuvent avoir en eux. Même si ce n'est pas de la pédagogie, cela a une influence directe sur la réussite scolaire ; même si c'est un contexte qui leur permet de travailler, si ce contexte est agréable, cela aura forcément des répercussions sur le plan scolaire.

Florence Bouteiller: Je ne suis pas partie, mais j'ai écouté ce que les collègues ont dit quand ils sont revenus et ce que j'ai retenu de ce qu'ils avaient dit, c'est qu'ils étaient très surpris de l'absence de contrôle par l'enseignant sur ce que fait l'élève. C'est-à-dire qu'on donne à l'élève une tâche à accomplir, mais s'il ne fait rien ou s'il fait mal, il n'y a pas d'intervention spécifique ou systématique de l'enseignant qui ne reprend pas la manière dont l'élève travaille. Et ça a été assez déstabilisant pour les collègues qui ont observé cela.

Yohann Aucante: Et est-ce que cela peut être repris par d'autres élèves dans le cadre d'un travail de groupe?

Virginie Delaunay (S) : En Suède particulièrement, on a observé pas mal de cours où le professeur était plutôt là comme une ressource disponible dans la classe pour les élèves. Les élèves avaient leur programme de travail au tableau dans beaucoup de cours, que ce soit au collège ou au lycée. La posture de contrôle de l'enseignant durait 10 minutes où il expliquait ce qui allait être fait pendant la séance, puis les enfants travaillaient soit individuellement soit en groupes parce qu'ils préféraient travailler à plusieurs. Il y avait beaucoup de systèmes d'autoévaluation, c'est-à-dire que dans les manuels ils ont des livrets avec les exercices déjà corrigés et ils s'auto-évaluent. Et les élèves n’allaient chercher le professeur que quand ils avaient vraiment besoin de son aide parce qu'ils n'avaient pas trouvé 
d'aide auprès des camarades, car la première personne à laquelle ils allaient se référer, c'était un camarade dans la classe, d'autant qu'ils se levaient assez librement.

Yohann Aucante: Et pour les plus petits niveaux d'âge ?

Virginie Delaunay $(S)$ : On en a observé un tout petit peu moins en primaire. Mais c'est vrai qu'il y a eu beaucoup de moments sur les séances que l'on a observées où les enfants travaillaient en autonomie sur une tâche donnée ; on avait le sentiment d'activités très ritualisées. On a vu une séance d'anglais par exemple, pour des élèves de CE1, et, de toute évidence, ils avaient acquis tout le déroulement de la séance sur d'autres séances antérieures ; ils savaient avancer tout seuls ; le seul rôle de l'enseignante consistait à leur dire quand changer d'activité.

Estelle Bucquet (D): Au Danemark, c'est vraiment l'école primaire que j'ai trouvé la plus déroutante parce que c'est là que l'autonomie et la responsabilisation étaient les plus développées. Les activités individuelles ou en groupes étaient très bien menées ; aucun élève n'était laissé de côté. En plus c'était du danois, donc on aurait pu croire à un phénomène de lassitude mais non, car c'était la même leçon travaillée différemment, d'abord de façon traditionnelle, puis sous forme ludique avec des élèves qui courent littéralement pour aller chercher les premiers des mots danois sur un tapis, en équipes. Puis on reprend ces mêmes activités avec une autocorrection, puis on retravaille en équipes. Cette alternance entre des activités et des temps impartis pour chacun que les élèves respectent systématiquement nous a vraiment fascinés.

Yohann Aucante : Est-ce que les outils numériques ou technologiques ont eu une place importante dans ce type de travail ?

Virginie Delaunay (S): Justement, dans cette séance d'anglais, la maîtresse était là simplement pour lancer les activités, mais il y avait le tableau numérique interactif, un jeu avec une roue à tourner et c'était un élève dans la classe qui devait faire tourner la roue avec le tableau : tout était géré par rapport à ce tableau numérique.

Hélène Perez (D) : Moi, j'étais déconcertée par la confiance qu'on faisait aussi aux enseignants puisqu'il n'y a pas ce système d'inspecteurs que nous avons ici en France et qui peut parfois être mal vécu. Dans l'école où nous étions au Danemark, il y avait une référente pédagogique qui la moitié du temps enseignait et l'autre moitié était là pour aider les collègues. On a du coup ressenti beaucoup plus de bienveillance entre collègues; si un enseignant est en difficulté avec une classe, il n’y a pas de jugement sur sa capacité à gérer, mais plutôt des conseils qui lui sont donnés pour modifier certaines choses. Cela nous a amenés à réfléchir à " Qu'est-ce qu'un bon cours? ?. Parce qu'en France, on a l'impression que le bon cours, c'est quand on passe dans une classe et qu'il n'y a pas un bruit ; or, en cours de langues, vu que c'est vivant, il y a du bruit, nécessairement, ce qui 
amène à un jugement de la part des collègues. Donc, personnellement, cela m'a décomplexée : ce n'est pas grave si je n'ai pas fait ma «trace écrite» en fin d'heure comme le recommandent les inspecteurs, je le ferai après; ce qui compte c'est que les élèves aient compris, tant pis s'ils n'ont pas écrit ou pas grand-chose, ça, on peut toujours le rattraper le lendemain.

Nicolas Buquet (D) : Je rejoins ce que dit Hélène sur la gestion du temps et le fait d'être moins stressé par le temps, le numérique prend son importance, car on peut enregistrer des choses et y revenir juste après. Quand on est sur un tableau-craie ou feutre, on écrit des choses et c'est difficile pour certains élèves quand l'enseignant dit " c'est terminé " et efface le tableau. Avec le numérique, c'est très facile de revenir en arrière et d'enregistrer ce qu'ont fait les élèves pour le reprendre plus tard. Dans le premier degré, l'utilisation du numérique n'est pas aussi démocratisée que dans le secondaire, quand un tableau est plein, qu'on a 21 élèves et qu'ils ne travaillent pas à la même vitesse, on est tenu par le temps en permanence. Personnellement j'ai été plus que surpris par la gestion du temps, particulièrement, comme disait Estelle, dans la classe de primaire danoise où rien n'est jamais grave. "Tu n'as pas terminé ? Ce n'est pas grave, on fera plus tard. » Alors que nous on passe notre temps à dire à des petits enfants de 6-7 ans : "Dépêche-toi! »

Yohann Aucante: Cette gestion du temps, on pourrait la projeter sur l'ensemble de la carrière de l'élève ?

Nicolas Buquet (D) : Complètement !

Virginie Delaunay (S): Mais visiblement l'importance de la trace écrite n'est pas la même que chez nous. Là-bas, il n'y avait pas beaucoup de traces écrites sur les tableaux, même en primaire. Il y avait en revanche beaucoup d'oral, ou de travail sur des jeux, de la manipulation, mais la trace écrite, la « leçon " d'histoire ou de géographie telle qu’on la conçoit, nous, on ne l'a pas observée.

Estelle Bucquet (D) : Dans la délégation danoise, si on a vraiment adhéré à l'utilisation du numérique en primaire, en revanche, on a émis de sérieuses réserves devant l'excès du numérique au lycée. On a eu l'occasion d'échanger avec les collègues sur place qui, eux-mêmes, reconnaissent que ça commence à leur poser problème. C'est beaucoup plus difficile de surveiller qu'un jeune derrière sa tablette n'est pas en train de faire sa commande sur Amazon - ce qui leur arrive apparemment - et surtout, tout dépend du numérique ; ce sont des jeunes qui arrivent au lycée sans feuille de papier, sans crayon ; tout repose intégralement sur le numérique, ce qui devient peut-être excessif. Autant il faut reconnaitre que c'est très bien dosé dans le primaire, autant au lycée, les collègues eux-mêmes commencent à revenir un peu sur le principe et à remettre en question l'exclusivité des tablettes. 
Maria Hellerstedt: Il y a des études scientifiques qui montrent que ce n'est pas bon.

Florence Bouteiller: Que cela n'améliore pas les performances des élèves.

Yohann Aucante : Oui, on peut les utiliser mais pas systématiquement.

Séverine Caron $(F)$ : Pour la Finlande, par rapport à l'évaluation des enseignants, nous avons eu l'occasion de discuter avec des responsables d'établissement et quand on leur posait la question de l'évaluation, ils étaient interloqués, ils ne comprenaient pas le sens de notre question. Ils nous expliquaient qu'ils faisaient une totale confiance aux professeurs qu'ils recrutaient et leur porte est toujours ouverte : ils préfèrent que ceux-ci viennent les voir s'ils sont dans le besoin, mais ils ne considèrent pas qu'ils ont à juger leur travail.

Audrey Techer (S) : Pour la Suède, ce qu'on a vu de déstabilisant pour les enseignants se trouvait particulièrement dans l'établissement privé Mikael Elias. C'est vrai qu'ils subissent énormément de pression, ils ont beaucoup de travail, en plus il n'y a pas de vie scolaire donc ils doivent gérer les élèves. Mais on a eu l'occasion d'interviewer des élèves et ils ont une relation à l'enseignant qui n'est pas du tout celle que nous avons en France, qui est plutôt quand même une relation d'hostilité de principe - un peu comme la relation entre enseignants et direction (je précise que je n'adhère pas à cet état de fait, je me contente de le décrire) - alors que là-bas, tout semble fondé sur la confiance et tout s'en passe beaucoup mieux, l'enseignant est un allié ; dès qu'un élève a un problème, c'est l'enseignant qu'il va voir, et comme il n'y a pas de $\mathrm{CPE}^{12}$, c'est lui qui va gérer le problème ; les familles sont en contact direct avec les enseignants, pour le meilleur ou pour le pire évidemment. Ici on a une vie scolaire, ça nous décharge d'un poids, mais d'un autre côté ça peut trop nous décharger au point de nous faire nous désintéresser, par exemple, de ce qui se passe dans le couloir dès qu'on sort de notre classe. Ce n'est pas bon non plus.

Yohann Aucante: La plus grande horizontalité des relations entre les différents acteurs du système joue peut-être, ainsi qu'une moins grande diversité des acteurs impliqués.

Hélène Perez (D) : Je suis plus ou moins d'accord. Ce n'est pas le fait qu'il y ait une vie scolaire qui empêche les élèves de venir voir les professeurs, je pense que c'est l'attitude de certains professeurs. Moi j'ai des élèves qui viennent me voir pendant la récréation, même des élèves que je n’ai pas cette année et je pense que tout dépend de la façon dont l'enseignant est avec ses élèves. S'ils voient que l'enseignant est bienveillant et s'intéresse vraiment à eux, ce n'est pas la présence d'une vie scolaire à côté qui les empêche de venir le voir.

12 Conseiller principal d'éducation. 
Dimitri Vaultier $(S)$ : Oui, mais c'est vrai qu'il y a souvent une posture de principe d'hostilité de la part de l'élève ou de l'enseignant.

Véronique Caijo : S’agissant du système français, CPE, personnel de direction, infirmière, assistante sociale ont des formations spécifiques que n'ont pas forcément les enseignants. En termes d'écoute, de secret partagé, de fonctionnement, d'accueil psychologique, parfois on peut avoir l'inverse de ce qu'il faudrait faire. Le tiers permet d'avoir du recul par rapport à certaines situations dangereuses. Je n'ai pas entendu de récit de situations dangereuses pour les élèves de la part des délégations, mais je pense qu'au Danemark, en Finlande et en Suède, il y a des élèves qui se suicident, des élèves qui ont des problèmes psychologiques... À un moment donné, peut-être que cet accompagnement-là qui existe en France permet d'avoir un regard un peu extérieur sur ce qui se passe dans la classe par rapport à l'enseignant, au relationnel de l'enseignant et de l'élève. Moi, j'y vois du bénéfice, parce que c'est plus facile peut-être d'être moins engagé affectivement que ce que les enseignants peuvent être.

Florence Lenois $(S)$ : Je pense qu'effectivement le fait qu'il y ait des personnes qui soient à l'écart et qui aient du recul aide à gérer certaines situations. En Suède, on a pu constater dans le grand lycée public de Varberg (Peder Skrivares) la présence de trois infirmières, trois psychologues et une assistante sociale à temps plein dans l'établissement, ce que nous n'avons pas en France.

Véronique Caijo : Ce qu’on n'a pas en France, donc le pôle psychologique de l'accompagnement de l'élève nous est dévolu à la vie scolaire. On n’a pas les moyens qu'eux déploient. Il faut remettre les choses en contexte.

Florence Lenois $(S)$ : Si l'enseignant était en difficulté ou s'il sentait qu'il y avait un mal-être chez certains élèves, il avait la possibilité de faire appel à ces personnes ressources de manière immédiate.

Véronique Caijo : Mais l'enseignant en France a aussi cette possibilité-là, sauf que les enseignants ne le font pas. Car on est aussi dans une distance hiérarchique qu'il n'y a pas forcément dans ces pays-là.

Séverine Caron $(F)$ : En Finlande, il y a le même système. Le collège était un établissement très récent avec une gestion de l'espace vraiment impressionnante ; le hall d'accueil était aussi un patio, qui était un vrai lieu de vie où se déroulaient les repas et il y avait une espèce de mezzanine avec une série de bureaux où se trouvaient infirmières, psychologues, médecins ; les familles entraient et sortaient pour venir rencontrer ces spécialistes avec un libre accès.

Yohann Aucante: De quelle manière pensez-vous intégrer d'autres choses à moyen terme dans vos pratiques et diffuser auprès de vos collègues et de l'institution les bénéfices de ce type d'expérience? 
Florence Lenois $(S)$ : Dans notre établissement, qui est un des collèges partenaires du projet, on a tous beaucoup échangé avec les collègues sur ce qu'on avait découvert pendant cette semaine en Suède ; dans un second temps, si on peut faire partager notre expérience de manière plus globale, ça pourra peut-être permettre de sélectionner ce qui sera prioritaire par rapport aux problématiques de l'établissement. En tout cas, ça intéresse tout le monde, y compris les agents avec lesquels j'ai échangé. Donc ça veut dire que tout ce qui relève de l'éducation et de l'enseignement intéresse tout le monde dans un établissement. Et je pense que si on veut que les choses se passent au mieux concernant le climat scolaire ou s'agissant de la bienveillance, il faut vraiment intégrer tout le monde.

Véronique Caijo : Dans le projet Erasmus tel qu'il a été monté, il y a une phase de restitution et de transfert. Il y a d'une part la validation via les corps d'inspection; les délégations se sont aussi engagées à transmettre l'expérience vécue et cela a déjà commencé, car l'inspecteur du premier degré a sollicité nos collègues professeurs d'école qui sont partis. Le travail qui est fait actuellement et que l'on va reprendre en début d'année scolaire suivante consistera dans la synthèse et la formalisation des données à échanger. Le proviseur (Dominique Procureur) a déjà des idées par rapport à cela et on avait déjà évoqué la question. Surtout, il faut que ce type de projet très lourd en investissement humain soit partagé dans les établissements. Par exemple, ce matin j'échangeais avec Béatrice Vallée (principale adjointe du collège Rachel-Salmona du Tréport, membre du consortium) qui part dans un autre établissement à la rentrée : elle sera la porte-parole de ce projet là-bas. Les gens en parleront, testeront des choses dans leur établissement et c'est ainsi que cela essaimera. Il faut donc s'interroger sur comment on diffuse, sur quel support, de quelle façon.

Nicolas Buquet (D) : C'est une des grandes difficultés qu'on rencontre. Je travaille sur la ville de Dieppe où il y a 21 écoles et quand on essaie de bouger les lignes en essayant d'avoir une réflexion sur la journée de l'enfant, son rythme - il faut effectivement y inviter les personnels autres qu'enseignants, comme disait Florence, comme les personnels de service - en souhaitant acheter du mobilier, cela implique la municipalité qui met un point d'arrêt à notre désir de changement en disant non à nos demandes. Il faut y croire et faire en sorte que cela puisse bouger, mais c'est très compliqué. Par exemple sur le sujet de la semaine scolaire - sujet qui me tient à cœur -, quand j'entends dire qu'on est sur 4,5 jours parce qu'on a voulu raccourcir la journée des enfants, puis qu'on nous laisse le choix et qu'on revient à la semaine de 4 jours, je suis effaré et je me demande si on réfléchit à l'intérêt de l'enfant; est-ce de la bienveillance à l'égard des enfants que de leur imposer des cours de 8 heures à 17 heures? Nous les premiers souhaitons des pauses quand on se réunit comme aujourd'hui! Et c'est là où ça va être difficile de faire avancer les choses. Je rejoins tout à fait $\mathrm{M}^{\mathrm{me}} \mathrm{Caijo}$, il faut absolument 
qu'on continue le projet. On risque peut-être de passer pour des farfelus ou des utopistes, mais il faut quand même essayer.

Estelle Bucquet (D) : On a toujours des réunions début juillet pour préparer la rentrée suivante dans les différents établissements ; on a notamment prévu de se réunir et d'en parler avec des collègues. Certains sont ouvertement intéressés, d'autres beaucoup moins, car je pense que dans leur esprit on veut changer, révolutionner alors que ça n’a jamais été le but. Nous, ce qu'on veut c'est échanger, discuter, en commençant tranquillement avec les collègues volontaires, de la manière dont on pourrait en faire profiter nos élèves, tout simplement, sachant qu'en effet, on ne peut pas changer les choses tout seuls. En cours de langues, les élèves d'une même classe travaillent parfois avec des professeurs différents. Les libertés accordées par les uns et refusées par les autres sont des sources potentielles de conflits. D'où la nécessité d'harmoniser et de se mettre d'accord. Pour finir, on espère aussi que le projet va se poursuivre dans tous les sens du terme parce que les Danois ont fait une demande Erasmus pour la France et attendent la réponse, et on espère bien les accueillir dans nos divers établissements l'année prochaine.

Véronique Caijo : Suite au passage d'un inspecteur général, on nous a aussi demandé d'écrire quelque chose avec pour vocation d'être diffusé dans la Lettre de l'éducation et autres publications.

\author{
Propos recueillis \\ par Yohann Aucante, \\ rédacteur en chef de Nordiques \\ et Maria Hellerstedt, \\ maîtresse de conférences \\ à l'université Lille III
}

